\section{Pharmacotherapy for mild hypertension}

\author{
Diana Diao, James M. Wright, David K. Cundiff, \\ Francois Gueyffier
}

The independent commentary was written by Agostinho Tavares

\section{ABSTRACT}

BACKGROUND: People with no previous cardiovascular events or cardiovascular disease represent a primary prevention population. The benefits and harms of treating mild hypertension in primary prevention patients are not known at present. This review examines the existing randomized controlled trial (RCT) evidence.

OBJECTIVE: Primary objective: To quantify the effects of antihypertensive drug therapy on mortality and morbidity in adults with mild hypertension (systolic blood pressure (BP) 140-159 $\mathrm{mmHg}$ and/or diastolic BP 90-99 $\mathrm{mmHg}$ ) and without cardiovascular disease.

METHODS:

Search: We searched CENTRAL (2011, Issue 1), MEDLINE (1948 to May 2011), EMBASE (1980 to May 2011) and reference lists of articles. The Cochrane Database of Systematic Reviews and the Database of Abstracts of Reviews of Effectiveness (DARE) were searched for previous reviews and meta-analyses of anti-hypertensive drug treatment compared to placebo or no treatment trials up until the end of 2011.

Selection criteria: RCTs of at least 1 year duration.

Data collection and analysis: The outcomes assessed were mortality, stroke, coronary heart disease (CHD), total cardiovascular events (CVS), and withdrawals due to adverse effects.

MAIN RESULTS: Of 11 RCTs identified 4 were included in this review, with 8,912 participants. Treatment for 4 to 5 years with antihypertensive drugs as compared to placebo did not reduce total mortality (RR 0.85, 95\% Cl $0.63,1.15)$. In 7,080 participants treatment with antihypertensive drugs as compared to placebo did not reduce coronary heart disease (RR 1.12, $95 \% \mathrm{Cl} 0.80,1.57$ ), stroke (RR 0.51, 95\% Cl 0.24, 1.08), or total cardiovascular events (RR 0.97, 95\% Cl 0.72, 1.32). Withdrawals due to adverse effects were increased by drug therapy (RR 4.80, 95\% Cl 4.14, 5.57), ARR 9\%.

AUTHORS' CONCLUSIONS: Antihypertensive drugs used in the treatment of adults (primary prevention) with mild hypertension (systolic BP 140-159 mmHg and/or diastolic BP 90-99 $\mathrm{mmHg}$ ) have not been shown to reduce mortality or morbidity in RCTs. Treatment caused $9 \%$ of patients to discontinue treatment due to adverse effects. More RCTs are needed in this prevalent population to know whether the benefits of treatment exceed the harms.

This is the abstract of a Cochrane Review published in the Cochrane Database of Systematic Reviews (CDSR) 2012, issue 8, DOI: 10.1002/14651858.CD006742.pub8 (http://onlinelibrary.wiley.com/ doi/10.1002/14651858.CD006742.pub2/abstract). For full citation and authors details see reference 1 .

The full text is freely available from: http://cochrane.bvsalud.org/cochrane/show.php?db=reviews \& $\mathrm{mfn}=4030 \& \mathrm{id}=C D 006742 \&$ lang $=$ pt\&d blang $=\& l i b=C O C \&$ print $=y e s \#$

\section{REFERENCE}

1. Diao D, Wright JM, Cundiff DK, Gueyffier F. Pharmacotherapy for mild hypertension. Cochrane Database Syst Rev. 2012;8:CD006742.

\section{COMMENTS}

The capacity to lower most hypertensive individuals' blood pressure already exists, with minimal adverse effects. However, the debate regarding therapy has shifted from whether lowering blood pressure is beneficial to the relative benefits and risks of individualized antihypertensive medications and their long-term effects on cardiovascular disease and chronic renal disease outcomes, especially for patients with mild hypertension.

On the basis of extensive epidemiological and observational data, cardiovascular risk progressively increases, starting from blood pressure levels as low as 115/75 mmHg.' On the other hand, some prospective trials $s^{2-5}$ have shown that the benefits from lowering blood pressure below 130/80 mmHg still appear uncertain in groups such as the elderly, those with coexisting cardiovascular disease, chronic renal disease or diabetes mellitus, and those with other forms of high cardiovascular risk. Furthermore, the ACCORD trial ${ }^{6}$ showed that the additional medication required to achieve lower blood pressure goals may be associated with additional adverse effects.

A study in the most recently published Cochrane Database of Systematic Reviews (2012) ${ }^{7}$ questioned the risks and harm of pharmacotherapy for mild hypertension among primary prevention patients, because such information is still unavailable for this particular group. Based on four randomized controlled trials, the authors ${ }^{7}$ pointed out that, in comparison with placebo, antihypertensive drugs administered to 8,912 participants did not reduce total mortality after four to five years of follow-up, and that in 7,080 participants, pharmacological treatment failed to reduce the incidence of coronary heart disease, stroke or total cardiovascular events, in comparison with placebo. Moreover, patient withdrawals due to side effects were substantially increased by drug therapy. From these conflicting data, two questions arise. Firstly, at what blood pressure level should patients be kept? Secondly, how should they be treated, especially those with mild hypertension?

In our opinion, it is almost impossible to develop a prospective trial comparing pharmacological therapy to placebo among mildly hypertensive patients undergoing primary prevention to answer the above questions. In order to have a number of cardiovascular events or deaths large enough to achieve statistical significance in this low-risk population, a huge number of individuals would need to be enrolled and followed up for decades. Moreover, leaving thousands of patients without treatment for such a long time would also be a matter of concern. Although such an approach might be considered to be overly conservative, the issues are complex and mixed information becomes confusing in clinical practice. Based on data from epidemiological and prospective studies, as mentioned earlier, we think that the most reasonable approach at present would be to have a general blood pressure goal of lower than 140/90 $\mathrm{mmHg}$ among mild and low-risk hypertensive individuals, and always to start with non-pharmacological therapy. For cases requiring lower targets or that are unresponsive to lifestyle modifications, the therapy should be individualized on the basis of clinical judgment.

Agostinho Tavares. Associate Professor and Head, Discipline of Nefrology, Universidade Federal de São Paulo — Escola Paulista de Medicina (Unifesp-EPM), São Paulo, Brazil.

\section{REFERENCES}

1. Lewington S, Clarke R, Qizilbash et al. Age-specific relevance of usual blood pressure to vascular mortality: a meta-analysis of individual data for one million adults in 61 prospective studies. Lancet. 2002;360(9349):1903-13. 
2. Tight blood pressure control and risk of macrovascular and microvascular complications in type 2 Diabetes. UKPDS 38. UK Prospective Diabetes Study Group. BMJ. 1998;317(7160):703-13.

3. Hansson L, Zanchetti A, Carruthers SG, et al. Effects of intensive blood-pressure lowering and low-dose aspirin in patients with hypertension: principal results of the Hypertension Optimal Treatment (HOT) randomized trial. HOT Study Group. Lancet. 1998;351(9118):1755-62.

4. Cooper-DeHoff RM, Gong Y, Handberg EM, et al. Tight blood pressure control and cardiovascular outcome among hypertensive patients with diabetes and coronary artery disease. JAMA. 2010;304(1):61-8.

5. Messerli FH, Mancia G, Conti CR, et al. Dogma disputed: can aggressively lowering blood pressure in hypertensive patients with coronary artery disease be dangerous? Ann Intern Med. 2006; 144(12):884-93.

6. ACCORD Study Group, Cushman WC, Evans GW, et al. Effects of intensive blood-pressure control in type 2 diabetes mellitus. N Engl J Med. 2010;362(17):1575-85.

7. Diao D, Wright JM, Cundiff DK, Gueyffier F. Pharmacotherapy for mild hypertension. Cochrane Database Syst Rev. 2012;8:CD006742. 